\title{
SUBNOTIFICAÇÃO DE ACIDENTES DE TRABALHO COM MATERIAL BIOLÓGICO DE TÉCNICOS DE ENFERMAGEM EM HOSPITAL UNIVERSITÁRIO
}

\section{UNDERREPORTING OF OCCUPATIONAL ACCIDENTS WITH BIOLOGICAL MATERIAL OF NURSING TECHNICIANS IN A UNIVERSITY HOSPITAL}

\section{SUBINFORME DE ACCIDENTES LABORALES CON MATERIAL BIOLÓGICO DE TÉCNICOS DE ENFERMERÍA EN UN HOSPITAL UNIVERSITARIO}

\author{
Katia Maria Rosa Vieira ${ }^{1}$ \\ Francisco Ubaldo Vieira $\mathrm{Jr}^{2}$ \\ Zélia Zilda Lourenço de Camargo Bittencourt ${ }^{3}$
}

\begin{abstract}
Como citar esse artigo: Vieira KMR, Vieira Jr FU, Bittencourt ZZLC. Subnotificação de acidentes de trabalho com material biológico de técnicos de enfermagem em hospital universitário. Rev baiana enferm. 2020;34:e37056.

Objetivo: quantificar a subnotificação e motivos do não registro dos acidentes de trabalho com material biológico de técnicos de enfermagem em hospital universitário. Método: pesquisa de corte transversal quantitativa com proporção amostral de 25\%. Participaram 275 profissionais de 9 unidades que responderam questionário sobre risco ocupacional. Resultados: foram relatados 747 acidentes, sendo $71 \%$ não notificados. As variáveis: horário de trabalho, unidades e tipos de exposição foram correlacionadas estatisticamente à subnotificação $(\mathrm{p}<0,05)$. Os motivos para não registro: "Quem trabalha na enfermagem sofre acidente com material biológico" apresentou 3,5 vezes mais chances de subnotificar acidente percutâneo com sangue; e "A saída pode sobrecarregar colegas de trabalho" teve 2,3 vezes mais chances de subnotificar acidente mucocutâneo. Conclusão: as subnotificações dos acidentes foram 2,4 vezes maiores que as notificações, com predomínio da exposição mucocutânea e os principais motivos foram relacionados à pouca percepção sobre o risco ocupacional e a sobrecarga de trabalho.
\end{abstract}

Descritores: Saúde do trabalhador. Riscos ocupacionais. Acidentes de trabalho. Enfermagem. Exposição ocupacional. Notificação.

Objective: to quantify the underreporting and reasons for not recording occupational accidents with biological material of nursing technicians in a university hospital. Method: quantitative cross-sectional research with a sample proportion of 25\%. Participants were 275 professionals from nine units who answered a questionnaire about occupational risk. Results: 747 accidents were reported, 71\% of which were not reported. The variables: working hours, units and types of exposure were statistically correlated with underreporting $(p<0.05)$. The reasons for not recording: "Nursing work leads to accidents with biological material" were 3.5 times more likely to underreport percutaneous blood accident; and "Leaving the unit can overload co-workers" was 2.3 times more likely to report mucocutaneous accident. Conclusion: the underreporting of accidents was 2.4 times higher than the notifications,

\footnotetext{
Enfermeira. Mestre em Saúde, Interdisciplinaridade e Reabilitação da Faculdade de Ciências Médicas da Universidade Estadual de Campinas. Campinas, São Paulo, Brasil. katia_rosa@terra.com.br. https://orcid.org/0000-0003-2988-7437.

Engenheiro mecânico. Doutor em Ciências Médicas. Professor do Instituto Federal de Educação, Ciências e Tecnologia de São Paulo, Campus Campinas. Campinas, São Paulo, Brasil. http://orcid.org/0000-0003-0419-6971.

3 Assistente Social. Mestra e Doutora em Saúde Coletiva. Professora da Universidade Estadual de Campinas. Campinas, São Paulo, Brasil. https://orcid.org/0000-00026796-55I5.
} 
with predominance of mucocutaneous exposure and the main reasons were related to the low perception of occupational risk and work overload.

Descriptors: Occupational health. Occupational risks. Accidents, occupational. Nursing. Occupational exposure. Notification.

Objetivo: cuantificar la sub-notificación y las razones para no registrar accidentes de trabajo con material biológico de técnicos de enfermería en un hospital universitario. Método: investigación transversal cuantitativa con una proporción de muestra del 25\%. Los participantes fueron 275 profesionales de nueve unidades que respondieron un cuestionario sobre el riesgo laboral. Resultados: se notificaron 747 accidentes, $71 \%$ de los cuales no fueron reportados. Las variables: horas de trabajo, unidades y tipos de exposición se correlacionaron estadísticamente con la sub-notificación ( $p<0.05)$. Las razones para no registrar: "Quienes trabajan en enfermería sufren accidentes con material biológico" fueron 3,5 veces más propensas a sub-reportar accidente de sangre percutáneo; $y$ "La producción puede sobrecargar a los compañeros de trabajo" era 2,3 veces más probable que notificara un accidente mucocutáneo. Conclusión: la sub-notificación de accidentes fue 2,4 veces mayor que las notificaciones, con predominio de la exposición mucocutánea y las principales razones estaban relacionadas con la baja percepción del riesgo laboral y la sobrecarga de trabajo.

Descriptores: Salud laboral. Riesgos laborales. Accidentes de trabajo. Enfermería. Exposición profesional. Notificación.

\section{Introdução}

O contexto do trabalho em instituições hospitalares, em que predominam novas tecnologias, necessita de enfermagem com domínio técnico, conhecimento científico e dimensionamento adequado de recursos humanos para a assistência de qualidade e segurança ${ }^{(1)}$.

A práxis da equipe de enfermagem requer cuidado aos pacientes durante 24 horas, proximidade física, realização de procedimentos de assistência direta de caráter invasivo, com uso de objetos perfurocortantes, o que propicia que no seu dia a dia ocorram diversos momentos de exposição a material biológico com possibilidade de acidentes de trabalho (AT) ${ }^{(2)}$.

A exposição ocupacional pode ocorrer via percutânea (picadas de agulhas ou objetos perfurocortantes) ou por contato direto com sangue ou fluidos orgânicos em mucosas e/ou pele não íntegra, sendo os vírus da imunodeficiência humana, hepatites $\mathrm{B}$ e C considerados os de maior risco para infecções ${ }^{(3)}$.

Em 2011, os hospitais dos Estados Unidos registraram 253.700 lesões e doenças relacionadas ao trabalho, com taxa de 6,8 para 100 trabalhadores, superior aos índices das indústrias de construção e manufatura, consideradas potencialmente perigosas e que apresentaram 4,3 e 3,9, respectivamente, para cada 100 trabalhadores $^{(4)}$.
No Brasil, em 2015, dentre as 20 ocupações com maior exposição a material biológico, foram registradas 47.292 ocorrências, sendo 49,6\% entre técnicos e auxiliares de enfermagem ${ }^{(5)}$.

A literatura evidencia que essa problemática está presente no cotidiano da enfermagem em diferentes países e continentes ${ }^{(6-9)}$, gera alto custo econômico e sofrimento psicossocial ao trabalhador ${ }^{(10)}$.

Tem-se, na atualidade, associação entre os AT com material biológico e sobrecarga de trabalho de técnicos de enfermagem, sendo o estresse considerado como preditor de risco para esses agravos ${ }^{(11)}$.

A notificação do AT é exigência legal e obrigatória para o empregador, que deve realizar o registro no protocolo de Comunicação de Acidente de Trabalho (CAT) para a Previdência Social, quando o trabalhador tiver o contrato regido pela Consolidação das Leis do Trabalho (CLT). Esta prática é fundamental, pois legitima direitos trabalhistas e previdenciários ${ }^{(12)}$. No caso de servidor público estatutário, o AT deve ser comunicado ao Departamento de Perícias Médicas do Estado (DPME) ${ }^{(13)}$.

Entretanto, a subnotificação dos ATs apresenta altos índices $^{(9,14)}$ e dificulta a promoção de ações corretivas, bem como de estratégias de intervenção que assegurem maior notificação nas organizações.

A notificação dos ATs é necessária quando há contato com sangue ou fluidos orgânicos 
potencialmente infectantes, tais como: sêmen, secreção vaginal, liquor, líquido sinovial, líquidos de serosas (pleural, peritoneal, pericárdico), líquido amniótico e fluidos não infectantes (suor, lágrimas, fezes, urina e saliva), quando contaminados com sangue ${ }^{(3)}$.

Para diminuir a exposição ocupacional e a transmissão de patógenos, foram adotadas recomendações internacionais denominadas "Precauções Padrão", sendo preconizado o uso de equipamentos de proteção individual, higienização das mãos, manuseio cuidadoso de objetos perfurocortantes e descarte em recipientes resistentes a perfuração ${ }^{(15)}$.

Dado a gravidade dos acidentes percutâneos, países como os Estados Unidos da América, Canadá, Brasil, Taiwan, Reino Unido e União Europeia promulgaram legislações sobre o uso de dispositivos de segurança para minimizar a ocorrência desses eventos ${ }^{(16)}$.

O conhecimento sobre a incidência de subnotificação de AT de técnicos de enfermagem com material biológico é fundamental, pois esta categoria representa um expressivo quantitativo da força de trabalho com exposição ocupacional nas instituições de saúde ${ }^{(5)}$. Este estudo propicia a obtenção de informações do cenário institucional e fornece subsídios para adoção de medidas de prevenção e proteção à saúde dos trabalhadores.

Diante da problemática, o objetivo deste estudo foi quantificar a subnotificação de AT com material biológico de técnicos de enfermagem e os respectivos motivos do não registro.

\section{Método}

Realizou-se um estudo transversal de natureza quantitativa, desenvolvido em um hospital público universitário de grande porte e alta complexidade, localizado em um município do interior do estado de São Paulo, no período de setembro a dezembro de 2017.

A amostra foi composta por técnicos de enfermagem; na ocasião da pesquisa a instituição possuía um quadro de 1.022 profissionais dessa categoria. Utilizou-se a proporção de aproximadamente $25 \%$, ficando a amostra constituída por 275 técnicos de enfermagem. A amostragem foi do tipo probabilística aleatória simples, com distribuição proporcional nas unidades e turnos de trabalho.

Foram convidados a participar da investigação, os profissionais das unidades: Internação Adulto (UIA) e Pediátrica (PED), Terapia Intensiva Adulto (UTI-ADU) e Pediátrica (UTI-PED), Emergência Referenciada (UER), Centro Cirúrgico (CC), Central de Material Esterilizado (CME), Imaginologia (IMA) e Serviço de Ambulatórios e Procedimentos Especializados (SEAMPE).

Adotou-se como critérios de elegibilidade os técnicos de enfermagem que realizavam assistência direta ou indireta aos pacientes da instituição nos turnos de trabalho: matutino (M) das 6h55 às 13h10, vespertino (V) das 13h às 19h15, e noturno $(\mathrm{N})$ das 19 h05 às 7h05. O SEAMPE realizava as atividades das $7 \mathrm{~h}$ às $18 \mathrm{~h} 30$, sendo neste estudo denominado horário administrativo (AD). Os critérios de exclusão foram: profissionais que estavam de folga, férias, licença médica, licença gestante e afastados por motivo de saúde.

Para o protocolo de estudo, elaborou-se um questionário com três blocos de perguntas estruturadas da seguinte forma:

Bloco 1: Relacionadas à caracterização socioeconômica e situação funcional;

Bloco 2: Relacionadas à ocorrência de AT com material biológico, notificados e subnotificados. O padrão de respostas foi estruturado em uma escala do tipo Likert com cinco opções: nenhum $=0 ; \mathrm{um}=1 ;$ dois $=2 ;$ três $=3$; quatro $=4 ;$ cinco ou mais $=5$;

Bloco 3: Relacionadas aos motivos para não notificação dos ATs com 15 opções de respostas, podendo ser escolhida mais de uma alternativa.

Os dados foram incluídos em planilha do MSExcel 2016 (Microsoft) e analisados no programa estatístico BioEstat 5.2. Utilizou-se o teste Qui-quadrado para análise da tabela de contingência, o teste Binomial para a comparação entre as proporções, e a Regressão Logística para análise combinada dos motivos para subnotificação. A consistência interna do questionário referente ao Bloco 2 foi avaliada pelo coeficiente alfa de Cronbach. Para todos os dados, os valores de $\mathrm{p}<0,05$ foram considerados como estatisticamente significantes. 
A pesquisa seguiu os preceitos éticos em conformidade com as diretrizes da Resolução no 466/2012 e foi aprovada pelo Comitê de Ética em Pesquisa da Universidade sob Parecer $\mathrm{n}^{-}$2.242.789/2017.

\section{Resultados}

Participaram da pesquisa 275 técnicos de enfermagem, com idades variando de 22 a 66 anos, com predomínio do sexo feminino $(83,6 \%)$, casados $(57,1 \%)$, ensino superior completo $(33,8 \%)$ e renda mensal aproximada de três a quatro salários mínimos (80,5\%). A maioria referiu ter somente um emprego (78,5\%), com jornada semanal de 30 horas $(77,8 \%)$ e vínculo trabalhista celetista $(46,9 \%)$.

O coeficiente do alfa de Cronbach foi calculado para o Bloco 2 e teve valor 0,65, sendo considerado de confiabilidade moderada.

A Tabela 1 mostra a distribuição dos ATs notificados e não notificados segundo as variáveis investigadas.

Tabela 1 - Distribuição do número de acidentes de trabalho notificados e não notificados segundo sexo, faixa etária, unidades, horário de trabalho e tipo de exposição ocupacional. Estado de São Paulo, Brasil - setembro a dezembro de $2017(\mathrm{~N}=747)$

\begin{tabular}{|c|c|c|c|c|c|}
\hline \multirow{3}{*}{ Variável } & \multicolumn{5}{|c|}{ Notificação Acidentes deTrabalho } \\
\hline & \multicolumn{2}{|c|}{ Sim (217) } & \multicolumn{2}{|c|}{ Não (530) } & \multirow{2}{*}{$\mathbf{p}^{*}$} \\
\hline & $\mathbf{n}$ & $\%$ & $\mathbf{n}$ & $\%$ & \\
\hline \multicolumn{6}{|l|}{ Sexo } \\
\hline Feminino & 170 & 28,2 & 432 & 71,8 & 0,37 \\
\hline Masculino & 47 & 32,4 & 98 & 67,6 & \\
\hline \multicolumn{6}{|l|}{ Faixa etária } \\
\hline 19 a 29 anos & 17 & 23,6 & 55 & 76,4 & 0,15 \\
\hline 30 a 39 anos & 56 & 25,0 & 168 & 75,0 & \\
\hline 40 a 49 anos & 81 & 32,9 & 165 & 67,1 & \\
\hline 50 a 59 anos & 60 & 32,1 & 127 & 67,9 & \\
\hline \multicolumn{6}{|l|}{ Unidades } \\
\hline Central de Material Esterilizado & 25 & 47,2 & 28 & 52,8 & 0,0001 \\
\hline Imaginologia & 21 & 70,0 & 9 & 30,0 & \\
\hline Centro Cirúrgico & 60 & 43,8 & 77 & 56,2 & \\
\hline Serviço de Ambulatórios e Procedimentos & 18 & 48,6 & 19 & 51,4 & \\
\hline Especializados & & & & & \\
\hline Pediatria & 10 & 16,9 & 49 & 83,1 & \\
\hline Unidade de Emergência Referenciada & 16 & 36,4 & 28 & 63,6 & \\
\hline Unidade de Terapia Intensiva Pediátrica & 1 & 4,3 & 22 & 95,7 & \\
\hline Unidade de Terapia Intensiva Adulto & 13 & 10,2 & 114 & 89,8 & \\
\hline Unidade de Internação Adulto & 53 & 22,4 & 184 & 77,6 & \\
\hline \multicolumn{6}{|l|}{ Horário de trabalho } \\
\hline Matutino & 83 & 33,6 & 164 & 66,4 & 0,0011 \\
\hline Vespertino & 59 & 29,4 & 142 & 70,6 & \\
\hline Noturno & 57 & 21,8 & 205 & 78,2 & \\
\hline Administrativo & 18 & 48,6 & 19 & 51,4 & \\
\hline \multicolumn{6}{|l|}{ Tipos de exposição } \\
\hline Percutânea com sangue & 90 & 65,7 & 47 & 34,3 & 0,0001 \\
\hline Percutânea sem sangue & 75 & 28,7 & 186 & 71,3 & \\
\hline Mucocutânea & 52 & 14,9 & 297 & 85,1 & \\
\hline
\end{tabular}

Fonte: Elaboração própria.

*Teste Qui-Quadrado. 
Os técnicos de enfermagem declararam ter sofrido 747 ATs com exposição a material biológico, sendo 217 notificados (29\%) e 530 não notificados (71\%), e a comparação entre as proporções mostrou diferenças estatísticas significantes $(\mathrm{p}<0,0001)$.

Dentre os participantes, $76 \%$ sofreram pelo menos um AT durante o tempo de vida laboral na instituição e 61\% declararam que tiveram pelo menos um AT não notificado.

Houve associação estatisticamente significante para subnotificação para as variáveis horário de trabalho, tipos de exposição ocupacional e unidades de trabalho. As maiores proporções de subnotificação ocorreram no turno noturno (78,2\%), na exposição mucocutânea $(85,1 \%)$, nas unidades de UTI-PED $(95,7 \%)$ e UTI-ADU (89,8\%).

O Quadro 1 mostra as respostas dos participantes quanto aos possíveis motivos para a não notificação dos ATs na instituição, podendo ser escolhido um ou mais motivos.

Quadro 1 - Distribuição de frequências das respostas relacionadas aos motivos para não notificação dos Acidentes de Trabalho. Estado de São Paulo, Brasil - setembro a dezembro de 2017 (N=1129)

\begin{tabular}{|c|l|c|c|}
\hline Motivo & \multicolumn{1}{|c|}{ Descrição } & n & \% \\
\hline 1 & Demora no atendimento pós-acidente & 174 & 15,4 \\
\hline 2 & Falta de tempo para sair da unidade & 130 & 11,5 \\
\hline 3 & A saída da unidade pode sobrecarregar os colegas de trabalho & 115 & 10,2 \\
\hline 4 & Excesso de trabalho & 115 & 10,2 \\
\hline 5 & $\begin{array}{l}\text { Culpabilização pelo não uso de equipamento de proteção individual } \\
\text { no acidente de trabalho }\end{array}$ & 109 & 9,7 \\
\hline 6 & Não sabe onde e como notificar & 96 & 8,5 \\
\hline 7 & Receio de ser culpado pelo acidente de trabalho & 80 & 7,1 \\
\hline 8 & Sorologias negativas do paciente em que houve o acidente & 58 & 5,1 \\
\hline 9 & Acidente leve não precisa ser informado e faz parte do trabalho & 55 & 4,9 \\
\hline 10 & Medo de ser punido(a) pela chefia & 47 & 4,2 \\
\hline 11 & Medicamento antirretroviral causa efeitos desagradáveis & 44 & 3,9 \\
\hline 12 & Medo da descoberta de alguma doença & 31 & 2,7 \\
\hline 13 & Quem trabalha na enfermagem sofre acidente com material biológico & 28 & 2,5 \\
\hline 14 & Impossibilidade de identificação do paciente com o qual o indivíduo & 25 & 2,2 \\
\hline 15 & Se acidentou & 1129 & 1,9 \\
\hline Total & Contrair alguma doença após acidente é difícil & 22 & \\
\hline
\end{tabular}

Fonte: Elaboração própria.

A Tabela 2 apresenta a associação dos motivos com registros de não notificação nos

diferentes tipos de exposição ocupacional.

Tabela 2 - Regressão logística dos motivos associados à não notificação por tipos de exposição ocupacional com material biológico. Estado de São Paulo, Brasil - setembro a dezembro de 2017

(continua)

\begin{tabular}{l|c|c|c}
\hline Tipo de exposição & p-valor & Odds Ratio & Intervalo de Confiança 95\% \\
\hline $\begin{array}{l}\text { Percutânea com sangue } \\
\text { Motivo } 13\end{array}$ & 0,04 & 3,5 & 1,03 a 11,85
\end{tabular}

\section{Percutânea sem sangue}

Sem motivos associados $\quad>0,05$ 
Tabela 2 - Regressão logística dos motivos associados à não notificação por tipos de exposição ocupacional com material biológico. Estado de São Paulo, Brasil - setembro a dezembro de 2017

(conclusão)

\begin{tabular}{l|c|c|c}
\hline Tipo de exposição & p-valor & Odds Ratio & Intervalo de Confiança 95\% \\
\hline Mucocutâneo & 0,0098 & 2,5 & 1,25 a 4,16 \\
Motivo 9 & 0,012 & 2,3 & 1,21 a 4,16 \\
Motivo 3 &
\end{tabular}

Fonte: Elaboração própria.

Como se observa na Tabela 2, somente os motivos 3, 9 e 13 apresentaram associação significante com a subnotificação $(\mathrm{p}<0,05)$.

A Regressão Logística (Tabela 2) revela que os técnicos de enfermagem que declararam "Quem trabalha na enfermagem sofre acidente com material biológico", tiveram 3,5 vezes mais chances de não notificar AT com exposição percutânea com sangue $(\mathrm{p}<0,04)$.

A subnotificação da exposição mucocutânea declarada pelos técnicos de enfermagem foi associada aos motivos "Acidente leve não precisa ser informado e faz parte do trabalho" e "A saída da unidade pode sobrecarregar os colegas de trabalho", os quais tiveram 2,5 e 2,3 vezes mais chances, respectivamente, de não notificação desses AT.

\section{Discussão}

Neste estudo, houve predomínio do sexo feminino, resultado que se assemelha a outros estudos $^{(7-9)}$, e embora, na atualidade, a enfermagem tenha amplo campo de atuação, historicamente a profissão é constituída por contingente expressivo de mulheres ${ }^{(17)}$.

O turno noturno apresentou a maior proporção de subnotificação, o que pode estar relacionado ao processo operacional. O profissional, quando se acidenta, é encaminhado à UER, e no primeiro dia útil, necessita procurar o serviço médico especializado, sendo que a demora foi o principal motivo elencado pelos participantes para a não notificação dos AT. Essa condição, aliada ao estresse e cansaço decorrente da extensa jornada laboral, parece ser desencorajadora, haja vista que a demora configura-se em importante barreira para a notificação ${ }^{(18)}$.

Esse resultado aponta para a necessidade de revisão do fluxo de atendimento e requer a participação dos gestores e dos trabalhadores como os envolvidos diretamente na situação, assumindo o protagonismo na defesa de sua saúde no trabalho ${ }^{(19)}$

Pesquisas evidenciaram que inadequações dos serviços, quadro insuficiente, excessiva carga mental podem provocar cansaço, déficit de atenção e resultar em AT ${ }^{(20-21)}$.

O cotidiano nas unidades de terapia intensiva é permeado por carga de trabalho ininterrupta, uso de tecnologias e requer uma equipe de enfermagem atuante, a qual congrega saberes individuais e coletivos ${ }^{(1)}$. $\mathrm{Na}$ presente investigação, a UTI-PED e a UTI-ADU apresentaram as maiores proporções de subnotificações, podendo ser sugestivo de sobrecarga de trabalho, o que dificulta a saída dos profissionais para notificação.

A subnotificação dos AT com exposição mucocutânea, com respingos de sangue e/ou outros fluidos orgânicos (297) foi 5,7 vezes maior que a notificação (52), podendo estar relacionada à crença de que essa ocorrência é comum na enfermagem (motivo 9).

Há estudos com resultados similares à presente investigação, com baixas taxas de notificação de AT com exposição mucocutânea ${ }^{(6,9,18)}$, e somente em um estudo ${ }^{(6)}$ foi identificada maior frequência de notificação para esse tipo de exposição laboral.

Tendo em vista que as crenças dos trabalhadores podem comprometer a percepção do risco ocupacional, a educação permanente, com capacitações periódicas, pode favorecer a adoção de comportamentos preventivos $^{(21-22)}$ e a adesão às Precauções Padrão.

Neste estudo, apenas a exposição percutânea com sangue teve proporção de notificação superior à não notificação. Pesquisas revelaram que os acidentes percutâneos foram os mais notificados ${ }^{(7,9,18,23)}$, 
sendo provavelmente relacionados ao estresse e ao medo de adquirir infecções ${ }^{(23) .}$

Os acidentes perfurocortantes representam grave problema e sua redução depende de investimentos em dispositivos de segurança. Embora o custo inicial seja dispendioso, este é diluído ao longo do tempo. Cabe ressaltar que o zelo pode favorecer a percepção do trabalhador sobre seu valor no trabalho ${ }^{(10)}$.

Embora a notificação propicie amparo legal ao trabalhador e legitime os direitos trabalhistas $^{(12)}$, a subnotificação dos acidentes consiste em obstáculo e requer esforços para a implementação de medidas de proteção e estratégias que viabilizem maior notificação.

Os motivos 3, 9 e 13 tiveram associação estatística com a subnotificação, sendo que a baixa percepção do risco, também foi encontrada em outros estudos $^{(6,9,18)}$. A atual pesquisa identificou que a insuficiência na percepção do risco é um aspecto determinante para a subnotificação.

Com relação ao acidente percutâneo, é sugestivo que os trabalhadores tenham atribuído pouca importância para sua ocorrência, que consideraram como de possibilidade remota ou improvável para infecções ${ }^{(18)}$.

No presente estudo, os técnicos de enfermagem apresentaram maior proporção de subnotificação na exposição mucocutânea, sendo indicativo de que subestimaram o risco ocupacional nesse tipo de exposição, aumentando os índices de subnotificações. Faz-se necessária a sensibilização dos profissionais ${ }^{(24)}$ sobre o risco potencial de aquisição de patógenos, decorrentes de quaisquer tipos de exposições ocupacionais ${ }^{(3)}$.

Devido à sobrecarga de trabalho, o profissional privilegiou o compromisso com a equipe e a assistência, em detrimento do seu próprio atendimento de saúde e notificação.

Apesar de alguns autores apontarem que o sentido do trabalho no século XXI seja pautado no empoderamento e na ascensão socioeconômica da enfermagem e não mais devotado à abnegação e ao sacrifício ${ }^{(25)}$, no presente estudo observou-se o contrário, prevaleceu o altruísmo nas relações de trabalho, pois mesmo com o avanço tecnológico, o ser humano constitui-se em elemento fundamental para a assistência ${ }^{(2)}$.
Os motivos declarados pelos técnicos de enfermagem para a não notificação dos acidentes com material biológico, como demora no atendimento $(15,4 \%)$, falta de tempo para sair da unidade $(11,5 \%)$, e excesso de trabalho $(10,2 \%)$, dentre outros, apresentaram percentuais relevantes, porém, no presente estudo, não foram correlacionados estatisticamente com subnotificação. Entretanto, devem ser levados em consideração no planejamento de ações corretivas, pois se configuram como pilares para a proteção da saúde do trabalhador.

É importante ressaltar que a pesquisa contou com a lembrança dos participantes, e em especial, aqueles acidentes considerados de menor relevância podem não ter sido lembrados, portanto os resultados podem estar subestimados.

\section{Conclusão}

Conclui-se que as subnotificações dos AT com material biológico de técnicos de enfermagem foram 2,4 vezes maiores que as notificações, com predomínio da exposição mucocutânea. As maiores proporções de subnotificações ocorreram nas unidades de UTI-PED e UTI-ADU e acometeram os profissionais que atuavam no turno noturno da instituição.

Os principais motivos para a subnotificação estão relacionados à pouca percepção sobre o risco ocupacional e à sobrecarga de trabalho a que são submetidos os trabalhadores diuturnamente no seu cotidiano. Ademais, os resultados indicaram que as estratégias preventivas adotadas são insuficientes e requer educação em serviço, com capacitações periódicas, para sensibilização ao risco biológico.

Sugere-se o desenvolvimento de novos estudos sobre a temática, com vistas à construção de medidas de prevenção para redução dos ATs e melhoria da qualidade de vida dos trabalhadores de enfermagem.

\section{Colaborações:}

1 - Concepção, projeto, análise e interpretação dos dados: Katia Maria Rosa Vieira; 
2 - Redação do artigo e revisão crítica relevante do conteúdo intelectual: Francisco Ubaldo Vieira Junior;

3 - Aprovação final da versão a ser publicada: Zélia Zilda Lourenço de Camargo Bittencourt.

\section{Referências}

1. Branco LLWV, Beleza LO, Luna AA. Carga de trabalho de enfermagem em UTI neonatal: aplicação da ferramenta Nursing workload in neonatal ICU: application of the nursing activities score tool. Rev Fund Care Online. 2017;9(1):144. DOI: 10.9789/2175-5361.2017. v9i1.144-151

2. Miranda FMD, Cruz EDA, Félix JCV, Kalinke LP, Mantovani MF, Sarquis LMM. Profile of Brazilian workers victims of occupational accidents with biological fluids. Rev Bras Enferm. 2017;70(5):1061-8. DOI: 10.1590/0034-71672016-0482

3. Brasil. Ministério da Saúde. Secretaria de Vigilância em Saúde. Departamento de Vigilância, Prevenção e Controle das Infecções Sexualmente Transmissíveis, do HIV/Aids e das Hepatites Virais. Profilaxia Pós-Exposição de Risco (PEP) à Infecção pelo HIV, IST e hepatites [Internet]. Brasília (DF); 2017 [cited 2019 Nov 10]. Available from: http:// www.aids.gov.br/pt-br/pub/2015/protocoloclinico-e-diretrizes-terapeuticas-para-profilaxiapos-exposicao-pep-de-risco

4. Occupational Safety and Health Administration. Facts about Hospital Worker Safety [Internet]. Washington, D.C. (EUA); 2013 [cited 2019 Oct 10]. Available from: https://www.osha.gov/dsg/ hospitals/documents/1.2_Factbook_508.pdf

5. Departamento Intersindical de Estatística e Estudos Socioeconômicos. Anuário da saúde do trabalhador 2015 [Internet]. São Paulo (SP); 2016 [cited 2019 Nov 26]. Available from: http://escola.dieese.org. br/escola/pdfs/anuario-saude-trabalhador

6. Nouetchognou JS, Ateudjieu J, Jemea B, Mbanya D. Accidental exposures to blood and body fluids among health care workers in a Referral Hospital of Cameroon. BMC Res Notes. 2016;9(94):1-6. DOI: 10.1186/s13104-016-1923-8

7. Zhang X, Gu Y, Cui M, Stallones L, Xiang H. Needlestick and sharps injuries among nurses at a teaching hospital in China. Workplace Health Saf. 2015;63(5):219-25. DOI: $10.1177 / 2165079915580035$
8. Uğurlu Z, karahan A, Ünlü $H$, Abbasoğlu A, Elbas NÖ, Işik SA, et al. The Effects of Workload and Working Conditions on Operating Room Nurses and Technicians. Workplace Health Safe. 2015;63(9):399-407. DOI: $10.1177 / 2165079915592281$

9. Chalya PL, Seni J, Mushi MF, Mirambo MM, Jaka H, Rambau PF, et al. Needle-stick injuries and splash exposures among health-care workers at a tertiary care hospital in north-western Tanzania. Tanzan J Health Res. 2015;17(2):1-15. DOI: 10.4314/thrb. v17i2.3

10. Mannocci A, De Carli G, Di Bari V, Saulle R, Unim B, Nicolotti $\mathrm{N}$, et al. How much do needlestick injuries cost? A systematic review of the economic evaluations of needlestick and sharps injuries among healthcare personnel. Infect Control Hosp Epidemiol. 2016;37(6):635-46. DOI: 10.1017/ice.2016.48

11. Vieira KMR, Vieira Junior FU, Bittencourt ZZLC. Nursing technicians: working conditions and accidents in a school hospital. J Nurs UFPE on line. 2019;13:e242224. DOI: 10.5205/ 1981-8963.2019.242224

12. Brasil. Lei no 8.213 , de 24 de julho de 1991. Dispõe sobre os Planos de Benefícios da Previdência Social e dá outras providências [Internet]. Diário Oficial da União. Brasília (DF); 1991 [cited 2019 Nov 15]. Available from: https://www2.camara. leg.br/legin/fed/lei/1991/lei-8213-24-julho-1991363650-publicacaooriginal-1-pl.html

13. Governo do Estado de São Paulo. Secretaria Estadual de Saúde. Servidor Público Estadual/ SES [Internet]. Cartilha temática 9. 2012(esp) [cited 2019 Jun 10]. Available from: http://www. saude.sp.gov.br/resources/crh/ggp/cartilhas/ acervo-cartilhas/cartilha9-servidorpublico.pdf

14. Carvalho DC, Rocha JC, Gimenes MCA, Santos EC, Valim MD. Work incidents with biological material in the nursing team of a hospital in Mid-Western Brazil. Esc Anna Nery. 2018;22(1):e20170140. DOI: 10.1590/2177-9465-ean-2017-0140

15. Garner JS. Guideline for isolation precautions in hospitals. Infect Control Hosp Epidemiol [Internet]. 1996;17(1):54-80. DOI: 10.1017/S0195941700006123

16. Cooke CE, Stephens JM. Clinical, economic, and humanistic burden of needlestick injuries in healthcare workers. Med Devices (Auckl). 2017;10:225-35. DOI: 10.2147/MDER.S140846 
17. Salimena AMO, Elias EA, Souza IEO, Vieira LB. Falatório e ocupação no cotidiano das profissionais de enfermagem no cuidado de si e do outro. Rev baiana enferm. 2016;30(1):316-24. DOI: $10.18471 /$ rbe.v1i1.14393

18. Ferreira MD, Pimenta FR, Facchin LT, Gir E, Canini SRMS. Subnotificação de acidentes biológicos pela enfermagem de um hospital universitário. Cienc Enferm [Internet]. 2015 [cited 2019 Dec 6];21(2):21-9. Available from: https:// scielo.conicyt.cl/pdf/cienf/v21n2/art_03.pdf

19. Strausz MC, Guilam MCR, Oliveira SS. A intervenção em saúde do trabalhador na perspectiva dos atores históricos do campo. Rev bras saúde ocup. 2019;44:e25. DOI: $10.1590 / 2317-6369000015118$

20. Sarsangi V, Salehiniya H, Hannani M, Marzalehs, MA, Abadi, YS, Honarjoo F, et al. Assessment of workload effect on nursing occupational accidents in hospitals of Kashan, Iran. Biomed Res Ther. 2017;4(8):1527-40. DOI: 10.15419/bmrat.v4i08.226

21. Santos SVM, Macedo FRM, Silva LA, Resck ZMR, Nogueira DA, Terra FS. Work accidents and self-esteem of nursing professional in hospital settings. Rev Latino-Am Enfermagem. 2017;25:e2872. DOI: 10.1590/1518-8345.1632.2872
22. Pereira-Ávila FMV, Estelle Michinov E, Luna TDC, Conde PS, Pereira-Caldeira NMV, Góes FGB. Standard precautions questionnaire: adaptação cultural e validação semântica para profissionais de saúde no Brasil. Cogitare enferm. 2019;24:e59014. DOI: $10.5380 /$ ce.v24i0.59014

23. Toska A, Saridi M, Wozniak G, Rekleiti M, Mouskou S, Souliotis K, et al. Incidence and frequency of mucocutaneous exposure and percutaneous injuries in Greek nurses: are they protected enough? Balkan Military Medical Review. 2014;17(4):120-5. DOI: 10.5455/bmmr.176132

24. Vieira KMR, Vieira Junior FU, Bittencourt ZZLC. Occupational accidents with biological material in a school hospital. Rev Bras Enferm [Internet]. 2019;72(3):772-8. DOI: 10.1590/00347167-2018-0630

25. Rodrigues AL, Barrichello A, Morin EM. Os sentidos do trabalho para profissionais de enfermagem: Um estudo multimétodos. Rev Adm Empresas. 2016;56(2):192-208. DOI: 10.1590/S0034759020160206

Recebido: 31 de maio de 2020

Aprovado: 4 de julho de 2020

Publicado: 17 de setembro de 2020

A Revista Baiana de Enfermagem utiliza a Licença Creative Commons - Atribuição-NãoComercial 4.0 Internacional. https://creativecommons.org/licenses/by-nc/4.0/ Este artigo é de acesso aberto distribuído sob os termos da Licença Creative Commons (CC BY-NC). Esta licença permite que outros remixem, adaptem e criem a partir do seu trabalho para fins não comerciais. Embora os novos trabalhos tenham de lhe atribuir o devido crédito e não possam ser usados para fins comerciais, os usuários não têm de licenciar esses trabalhos derivados sob os mesmos termos. 